\title{
Constraints on the topology of the Universe: Extension to general geometries
}

\author{
Pascal M. Vaudrevange, ${ }^{1,2}$ Glenn D. Starkman, ${ }^{3}$ Neil J. Cornish, ${ }^{4}$ and David N. Spergel ${ }^{5}$ \\ ${ }^{1}$ DESY, Notkestrasse 85, 22607 Hamburg, Germany \\ ${ }^{2}$ CERCA and Department of Physics, Case Western Reserve University, 10900 Euclid Avenue, Cleveland, Ohio 44106, USA \\ ${ }^{3}$ CERCA, ISO and Department of Physics, Case Western Reserve University, 10900 Euclid Avenue, Cleveland, Ohio 44106, USA \\ ${ }^{4}$ Department of Physics, Montana State University, Bozeman, Montana 59717, USA \\ ${ }^{5}$ Department of Astrophysical Sciences, Princeton University, Princeton, New Jersey 08544, USA
}

(Received 25 June 2012; published 15 October 2012)

\begin{abstract}
We present an update to the search for a nontrivial topology of the Universe by searching for matching circle pairs in the cosmic microwave background [N. J. Cornish et al. Phys. Rev. Lett. 92, 201302 (2004)] using the WMAP 7-year data release. We extend the existing bounds to encompass a wider range of possible topologies by searching for matching circle pairs with opening angles $10^{\circ} \leq \alpha \leq 90^{\circ}$ and separation angles $11^{\circ} \leq \theta \leq 180^{\circ}$. The extended search reveals two small anomalous regions in the cosmic microwave background sky. Numerous pairs of well-matched circles are found where both circles pass through one or the other of those regions. As this is not the signature of any known manifold, but is a likely consequence of contamination in those sky regions, we repeat the search excluding circle pairs where both pass through either of the two regions. We then find no statistically significant pairs of matched circles, and so no hints of a nontrivial topology. The absence of matched circles increases the lower limit on the length of the shortest closed null geodesic that self-intersects at our location in the Universe (equivalently the injectivity radius at our location) to $98.5 \%$ of the diameter of the last scattering surface or approximately $26 \mathrm{Gpc}$. It extends the limit to any manifolds in which the intersecting arcs of said geodesic form an angle greater than $10^{\circ}$.
\end{abstract}

DOI: 10.1103/PhysRevD.86.083526

PACS numbers: 98.80.-k, 04.20.Gz, 98.70.Vc, 98.80.Es

\section{INTRODUCTION}

The search for a nontrivial topology of the Universe has enjoyed a long and fascinating history. Using different methods-from searching for specific topologies to the more general circles in the sky approach [1] - the cosmic microwave background (CMB) has been analyzed extensively, looking for any signs that light from the same object reaches us by more than one path (see Ref. [2] for a review of the various suggested methods, including Refs. [1,3-24]). So far, all specialized efforts to detect specific topologies as well as the search for matching opposing circles in the sky have failed to detect any sign of a nontrivial topology of our Universe.

The circles-in-the-sky method, which we adopt in this paper, is based on the following intuitive picture. For illustrative purposes, assume that the true topology of the Universe is a 3-torus, with unit cell size smaller than the Hubble horizon (see Fig. 1). This can be thought of as a tiling of flat space by identical cubes. An observer, such as ourselves, performing a series of CMB observations somewhere in one of the cubes, has clones identically located in each of the other cubes performing the identical series of observations. Centered around the observer is the 2 -sphere of the surface of last scattering at $z \approx 1100$, with CMB fluctuations imprinted on it. Around the clone of the observer on the right, there is another 2-sphere of the surface of last scattering. The intersection of both 2spheres is given by a circle. Both observers will look at the same ring of temperature fluctuations-albeit from different "sides." Both observers are in fact identical, so an observer will see a matching pair of circles: one to the left and to the right. Hence, comparing temperature fluctuations along circles potentially yields information about the topology of our Universe. Going away from toroidal geometries, it becomes immediately clear that the separation angle $\theta$ (the angle between the centers of the pair of matching circles) need not be $180^{\circ}$. Also, depending on the orientability or nonorientability of the manifold, circle pairs might have matching temperature fluctuations either both going clockwise around the

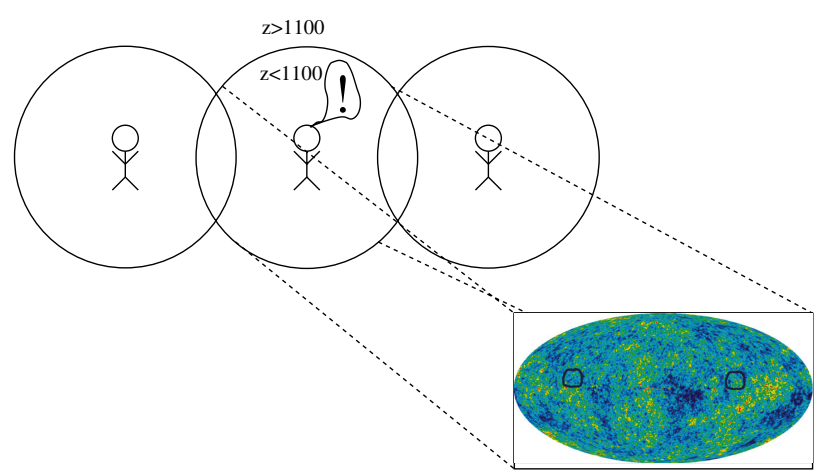

FIG. 1 (color online). Schematic geometry of the circlesin-the-sky method. If the topology of the Universe is a torus, the observer, located at the center of the 2-sphere of the surface of last scattering, sees matching circles of temperature fluctuations on opposite sides of the CMB sky. 
circles (nonorientable) or one going clockwise and the other anticlockwise (orientable).

So far, the search for matching antipodal circles, i.e., circles with separation angles $180^{\circ}$, or nearly antipodal circles [3], like other topology searches, has only yielded lower limits on the size of the Universe, and then only for "nearly flat" topologies.

In this work, we apply the circles-in-the-sky statistics to searches for circles pairs of all opening angles $10^{\circ} \leq$ $\alpha \leq 90^{\circ}$, and integer separation angles $11^{\circ} \leq \theta \leq 180^{\circ}$ with both orientations. This extends the previous searches $[3,6]$ to cover almost all possible topologies. We find what seems to be a systematic effect at two special positions in the sky that produces spurious signals for osculating circles. Otherwise, we see no evidence of nontrivial topology.

\section{METHODOLOGY}

As already outlined in the Introduction, the most intuitive way to search for a nontrivial topology of the Universe is by looking for matching pairs of circular temperature fluctuation patterns in the CMB.

In order to determine the underlying topology of the Universe, one would need to scan the full CMB map-at a Healpix ${ }^{1}$ resolution of $N_{\text {side }}=512$ corresponding to $3 \times 10^{6}$ pixels - for matching circle pairs. To conduct a search for all topologies, i.e., over all opening angles of the circles and all possible separation angles, an enormous number of circle pairs would need to be analyzed. Previously reported searches focused on nearly flat geometries, where matching circle pairs are at almost opposite positions on the sky, i.e., $\sim 180^{\circ}$ apart (see Refs. $[3,6]$ ).

Thanks to increasing computing power, we can now take a more general approach. In order to obtain an acceptable time frame for completing this project, we superimposed a search grid of $N_{\text {side }}=128$ onto the map, resulting in $2 \times 10^{10}$ circle pairs that need to be compared. We compute the goodness of the match for a given circle pair on the full resolution, $N_{\text {side }}=512$, map. Using a resolution of 512 pixels along a given circle costs about $10^{4}$ operations per compared pair, leading to a total number of operations of $2 \times 10^{14}$ per opening angle. On a $3 \mathrm{GHz} \mathrm{CPU}$, this takes about $20 \mathrm{hr}$ for a single opening angle. Scanning over 200 opening angles corresponds to $4000 \mathrm{CPU} \mathrm{hr}$, easily feasible on modern computer clusters.

Thus we search for all possible topologies, albeit on a somewhat lower resolution grid. Note that we need to run the search twice, once for orientable manifolds and once for nonorientable manifolds, i.e., once for circle pairs that are oppositely oriented, and once for circle pairs that are oriented in the same way.

A first Ansatz for a circle statistic $S_{i j}$ to measure the match between circles $i, j$ would be the convolution of the

\footnotetext{
${ }^{1}$ http://healpix.jpl.nasa.gov
}

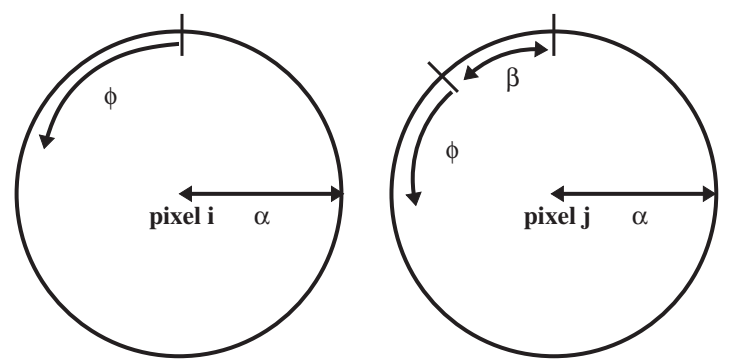

FIG. 2. A circle of opening angle $\alpha$ centered at pixel $i$, and a circle centered at pixel $i$ with its phase shifted by $\beta$.

temperature fluctuations along two circles. As discussed in Ref. [6], this would lead to a dominance of long wavelength (small $m$ ) Fourier modes along the circles, making $S_{i j}$ rather insensitive to small-scale fluctuations. To compensate for this, the authors of Ref. [3] introduced an additional factor of the wave number $m$ in the convolution (this is equivalent to the usual factor of $\ell(\ell+1)$ used to scale the two-dimensional power spectrum). Thus, in order to compare two circles of opening angle $\alpha$ centered around pixel numbers $i$ and $j$, we employ the circle statistic

$$
\begin{aligned}
S_{i j}(\alpha, \beta)= & \frac{1}{\sum_{m=0}^{n / 2} m\left(\left|T_{i m}(\alpha)\right|^{2}+\left|T_{j m}(\alpha)\right|^{2}\right)} \\
& \times\left(\sum_{m=0}^{n / 2} m T_{i m}^{*}(\alpha) T_{j m}(\alpha) e^{-\frac{2 \pi i m \beta}{n}}\right. \\
& \left.+\sum_{m=0}^{n / 2}(n-m) T_{i m}^{*}(\alpha) T_{j m}(\alpha) e^{-\frac{2 \pi i m \beta}{n}}\right),
\end{aligned}
$$

where $\beta$ is the relative phase between the two circles and $T_{i m}(\alpha)$ is the Fourier transform of the temperature fluctuation $\frac{\Delta T}{T}$ around circle $i$

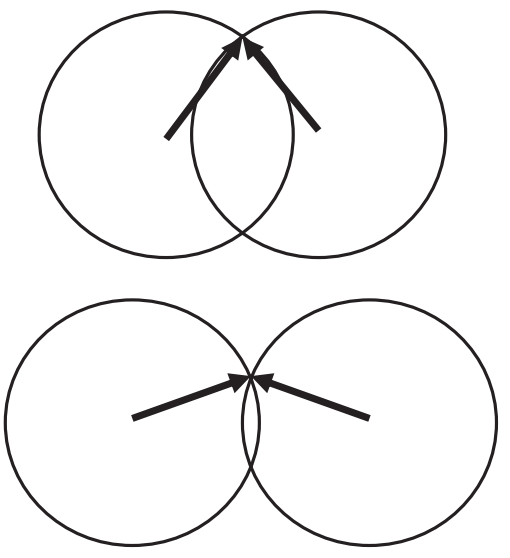

FIG. 3. Doppler contribution to the matching circle signal. Small circles (bottom) have opposite Doppler contributions, whereas large circles (top) obtain similar Doppler contributions. Thus large circles show a smaller deviation from $S_{i j}^{\max }=1$. 


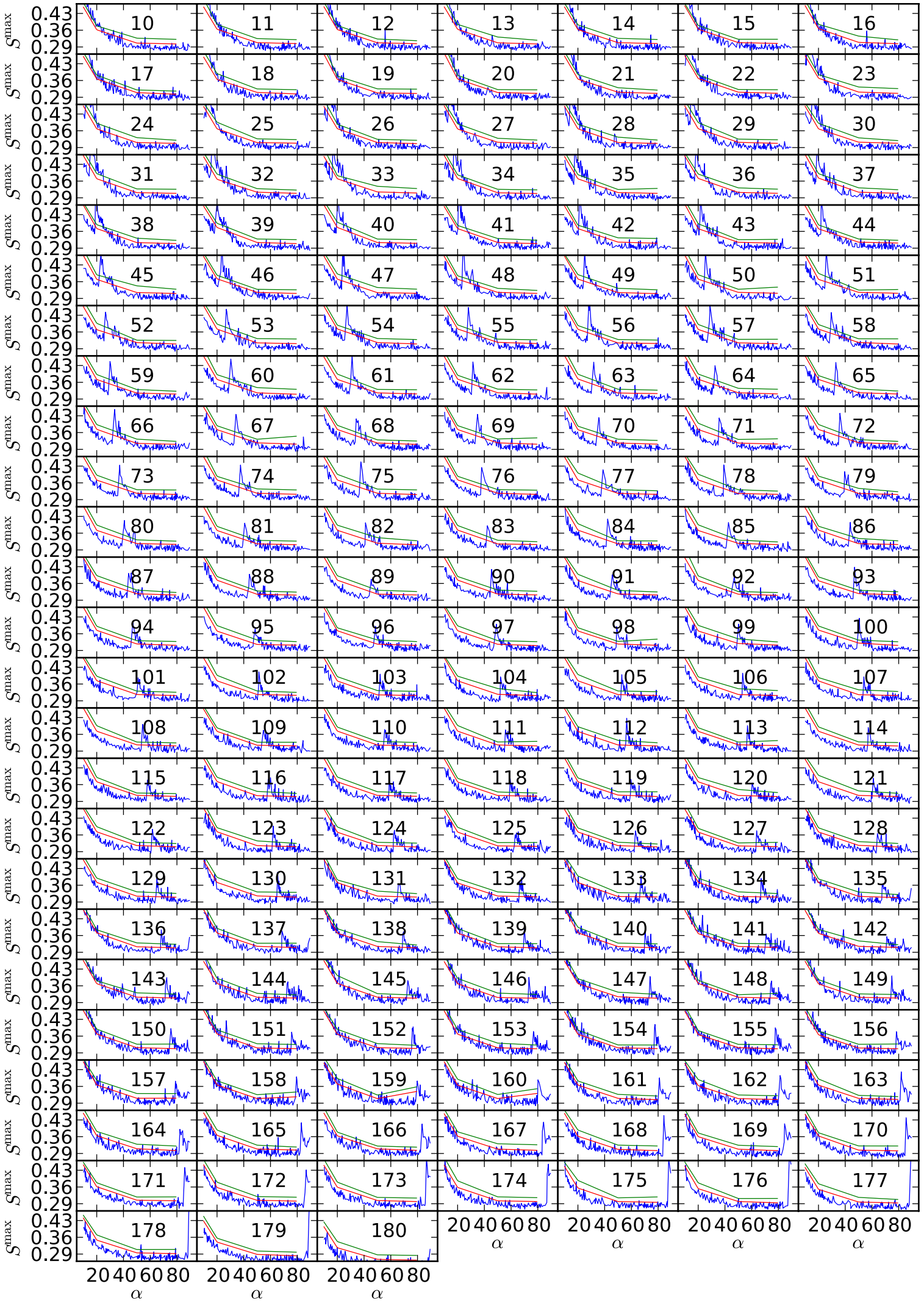

FIG. 4 (color online). Searching for orientable topologies: The statistics $S$ (solid blue line) as defined in Eq. (1) as a function of opening angle $\alpha$ and separation angle $\theta$ (indicated by the numbers in the center of each plot). The red (green) line corresponds to the $2 \sigma(3 \sigma)$ C.L. The spikes come from circles osculating at the galactic anticenter and at $l \approx 29^{\circ}, b \approx-19^{\circ}$ (see Fig. 7). Removing circles that touch these regions removes all features (see Fig. 8). 


$$
\frac{\Delta T}{T}(i, \phi)=\sum_{m=0}^{n-1} T_{i m} e^{\frac{2 \pi i m \phi}{n}},
$$

where $n=512$ along the circle (see Fig. 2). For a search for orientable manifolds, we replace all occurrences of $T_{i m}^{*}(\alpha)$ in the numerator by $T_{i m}(\alpha)$. Notice that we use the conventions of the LIBFFTW package $[25]^{2}$ : positive frequencies are stored in the first half of the array $T_{i m}, m=$ $0 \ldots \frac{n}{2}$, and negative frequencies are stored in backwards order from the end of the array, making $T_{i m}=T_{i(n-m)}^{*}{ }^{3}$ We then use as statistic the maximum of $S_{i k}(\alpha, \beta)$ over all relative phases $\beta$

$$
S_{i j}^{\max }(\alpha) \equiv \max _{\beta} S_{i j}(\alpha, \beta) .
$$

For perfectly matching circles, $S_{i j}^{\max }(\alpha)=1$, and for perfectly uncorrelated circles, $S_{i j}^{\max }(\alpha) \approx 0$. In practice, these ideal values are not realized due to noise contribution from several different effects. First of all, the Doppler effect at the surface of last scattering creates a different signal depending on the position of the observer, making larger matching circles closer to $S_{i j}^{\max } \approx 1$ whereas smaller circles will have $S_{i j}^{\max }<1$ (see Fig. 3). Another contribution comes from line-of-sight effects, especially the integrated Sachs-Wolfe effect, as the CMB photons from different directions traverse through different patches of space. The combination of these effects reduces the signal in $S_{i j}^{\max }$, making potential matching circles less than perfect (see Fig. 1 in Ref. [3]).

For this analysis, we used the WMAP7 temperature maps [26]. Outside the WMAP Kp $12^{4}$ sky mask, we used the same template cleaning method as Ref. [27]. Inside the Kp12 sky cut, we used the WMAP ILC map. ${ }^{5}$ While this choice implied that the noise properties and resolution of the map differed between the two regions, the effect on the circle search was relatively small as the internal linear combination (ILC) map was only used for $5.8 \%$ of the pixels.

In order to estimate the significance of potential spikes in the statistics, we created approximate random realizations of the CMB. ${ }^{6}$ To this end, we compute the $a_{\ell m}$ 's of the cleaned CMB map. Then, we scramble them by randomly interchanging the $m$ index for fixed $\ell$ 's and compute a "random" CMB map from the scrambled $a_{\ell m}$ 's. Using

\footnotetext{
${ }^{2}$ http://www.fftw.org

${ }^{3}$ Using the real-to-complex and complex-to-real routines enables us to save the Fourier transforms for a single circle of a given opening angle in an complex array of length $\frac{n}{2}$ instead of $n$. ${ }^{4} \mathrm{http}: / /$ lambda.gsfc.nasa.gov/product/map/dr1/intensity_mask .cfm

${ }^{5}$ http://lambda.gsfc.nasa.gov/product/map/dr4/ilc_map_get .cfm

${ }^{6}$ In contrast to the WMAP 1-year data that was examined in Ref. [3], here the statistics are not noise dominated. In particular, this means that the estimator for the false detection rate devised in Ref. [3] is not applicable for the WMAP 7-year data.
}

this map, we compute the statistics for matching (non) orientable circle pairs for opening angles $\alpha=20^{\circ}, 50^{\circ}$, $80^{\circ}$ and all integer separation angles $\theta$. Repeating this 1000 times, we obtain an estimate of the probability density function of $S^{\max }$. While there are enough samples to give a reliable estimate of the $95.4 \%(2 \sigma)$ confidence level (C.L.), the quoted $99.7 \%(3 \sigma)$ C.L. is at most a rough estimate. If the sky noise were isotropic, then this randomization process would generate simulated maps with the same statistical properties as the WMAP observations. However, because of anisotropies due to spatial variations in the WMAP noise (which is larger near the ecliptic plane) and variations in the resolution of our map (due to the need to use the ILC map in the galactic plane), these simulated maps only approximate the WMAP sky maps.

\section{RESULTS}

Implementing the procedure outlined in the previous section, we present the results of the searches and describe the systematic effects we encountered, both for orientable and nonorientable topologies.

Apart from a possible signal, there are simple random statistical fluctuations that are expected to exceed the 95\% C.L. To estimate the number of random fluctuations, we note that this can be viewed as a series of Bernoulli trials with probability $p=0.05$ for success, i.e., for a spike above $95 \%$ C.L. Per separation angle $\theta$, we probed $n=190$ values of the opening angle $\alpha$ (by choosing it to

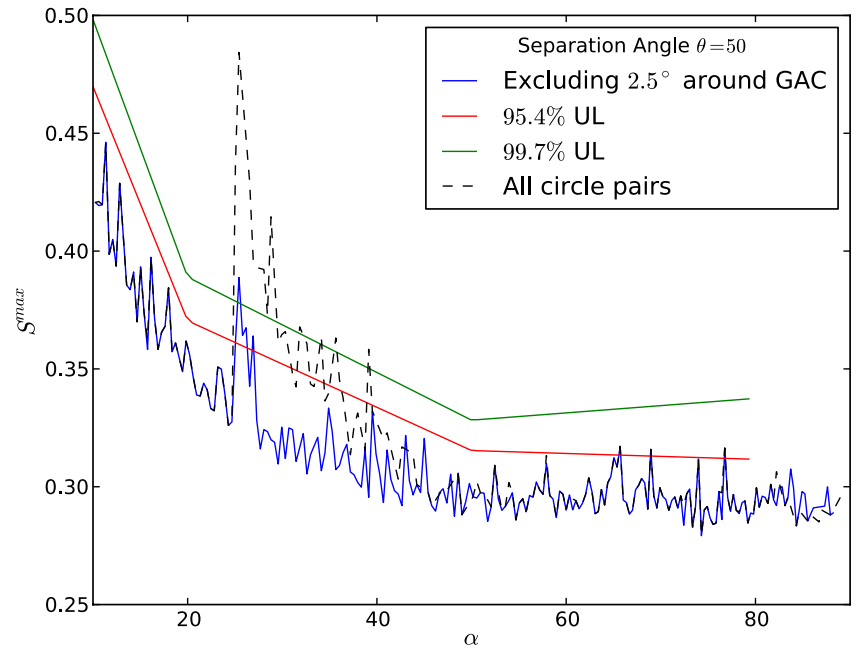

FIG. 5 (color online). Searching for orientable topologies: For separation angle $\theta=50^{\circ}$, a plot of the statistics $S$ as a function of opening angle $\alpha$. Without cutting anything, the dashed black line shows an extended feature at $\alpha=\frac{1}{2} \theta=25^{\circ}$. Ignoring all circle pairs that overlap within $2.5^{\circ}$ of the galactic anticenter (GAC), the feature mostly disappears (solid blue line). The red (green) solid line shows the $95.4 \%(99.7 \%)$ C.L. on $S$, obtained by scrambling the $a_{\ell m}$ (see the main text). See Fig. 7 for an explanation of the systematic spikes in $S$ as well as Fig. 8 for the final result after excising the offending circles. 


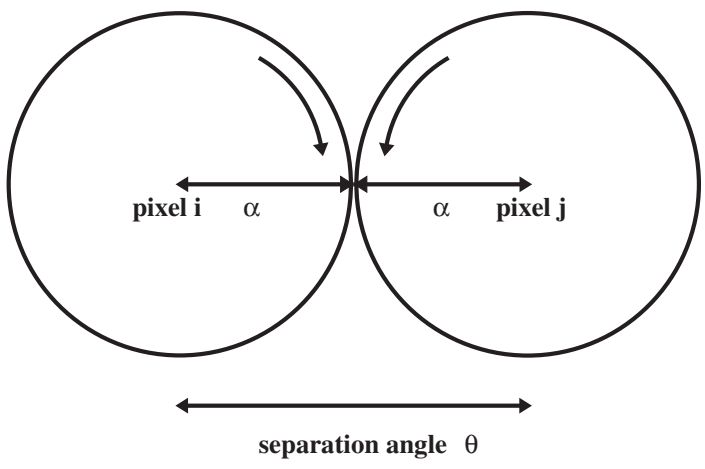

FIG. 6. As soon as the separation angle $\theta$ between the two circles becomes approximately twice their opening angle $\alpha$, $\theta<2 \alpha$, the circles start partially overlapping, creating matching patterns in the statistics $S^{\max }$ as for orientable manifolds; the "kissing" segments match.

lie on a grid deriving from the position of the rings in the Healpix scheme for $n_{\text {side }}=128$. The probability distribution function for having $i$ excursions above the $2 \sigma / 95.4 \%$ C. L. for a sequence of $n$ Bernoulli trials is given by the Binomial distribution

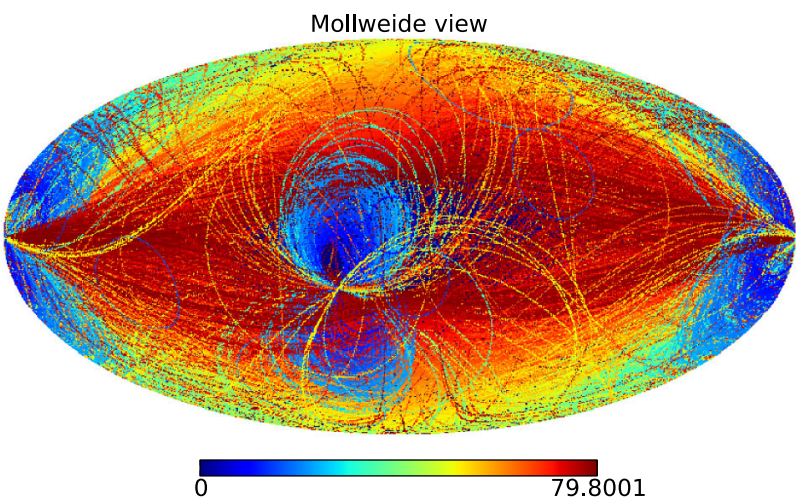

a)

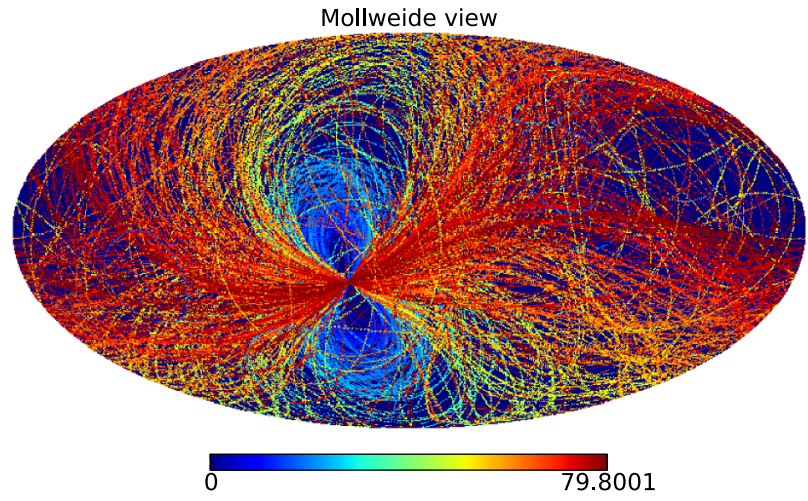

c)

FIG. 7 (color online). For the search for orientable manifolds: Location of the circles pairs with maximal statistics $S$ that lies above the $3 \sigma$ C.L., colored by opening angle $\alpha$. Note that the osculating circles at the galactic anticenter and at $l \approx 29^{\circ}, b \approx-19^{\circ}$ do not hint at a nontrivial topology. (a) The highest signal comes from circle pairs osculating at the galactic anticenter and at $l \approx 29^{\circ}$, $b \approx-19^{\circ}$. (b) Same as (a) but rotated by $180^{\circ}$ such that the galactic anticenter is in the middle of the plot. (c) Removing circle pairs that osculate at the galactic anticenter, the highest signal comes from pairs that osculate at $l \approx 29^{\circ}, b \approx-19^{\circ}$. (d) Removing circles that osculate either at the galactic anticenter or at $l \approx 29^{\circ}, b \approx-19^{\circ}$, no special position on the sky is apparent. 


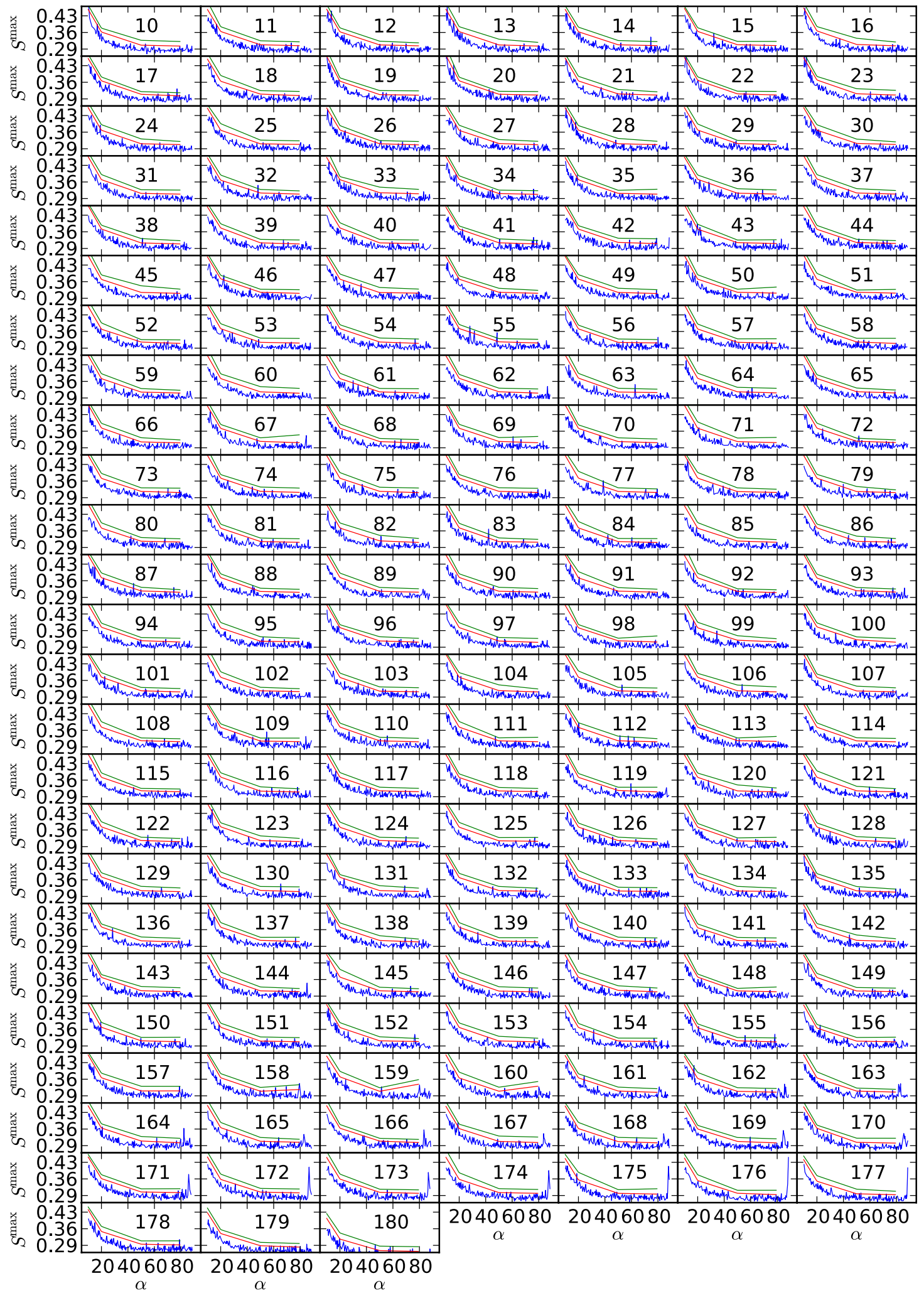

FIG. 8 (color online). Searching for orientable topologies, final result: The statistics $S$ (solid blue line) as defined in Eq. (1) as a function of opening angle $\alpha$ and separation angle $\theta$ (indicated by the numbers in the center of each plot). The red (green) line corresponds to the $2 \sigma(3 \sigma)$ C.L. We disregard circles that touch either the galactic anticenter or $l \approx 29^{\circ}, b \approx-19^{\circ}$. The peaks for separation angles $\theta>170^{\circ}$ and opening angle $\alpha \approx 90^{\circ}$ are caused by the fact that circles with $\alpha=90^{\circ}$ have an angular diameter of $180^{\circ}$. Thus, two such circles, when separated by $\theta \approx 180^{\circ}$ will start overlapping, until for $\theta=180^{\circ}$, they coincide, independent of the topology of the Universe. No signs of a nontrivial orientable topology are found. 


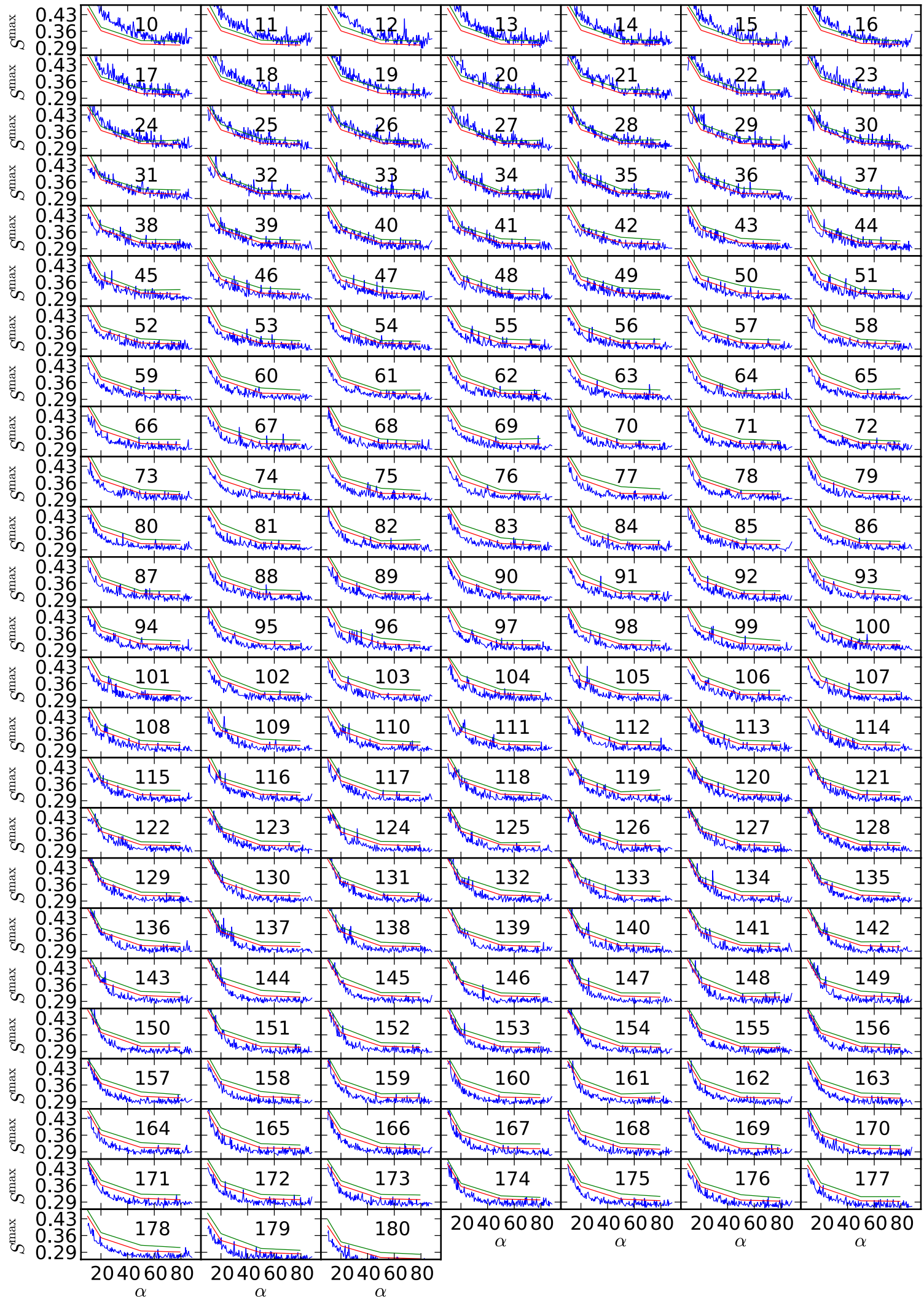

FIG. 9 (color online). Searching for nonorientable topologies: The statistics $S$ (solid blue line) as defined in Eq. (1) as a function of opening angle $\alpha$ and separation angle $\theta$ (indicated by the numbers in the center of each plot). The red (green) line corresponds to the $2 \sigma(3 \sigma)$ C.L. The spikes come from circles osculating in the galactic anticenter and at $l \approx 29^{\circ}, b \approx-19^{\circ}$ (see Fig. 10). Removing circles that touch these regions removes all features (see Fig. 11). 


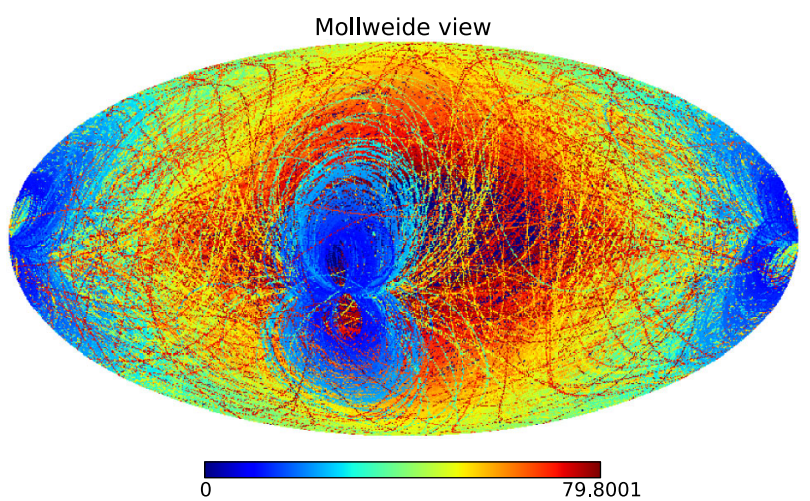

a)

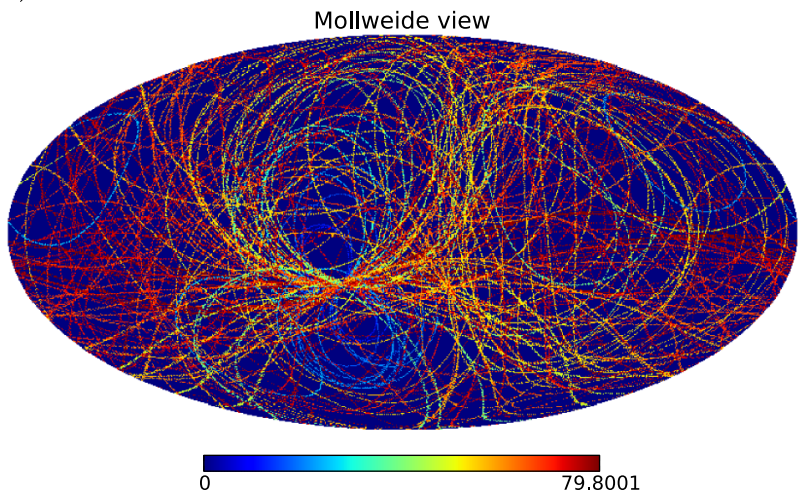

c)

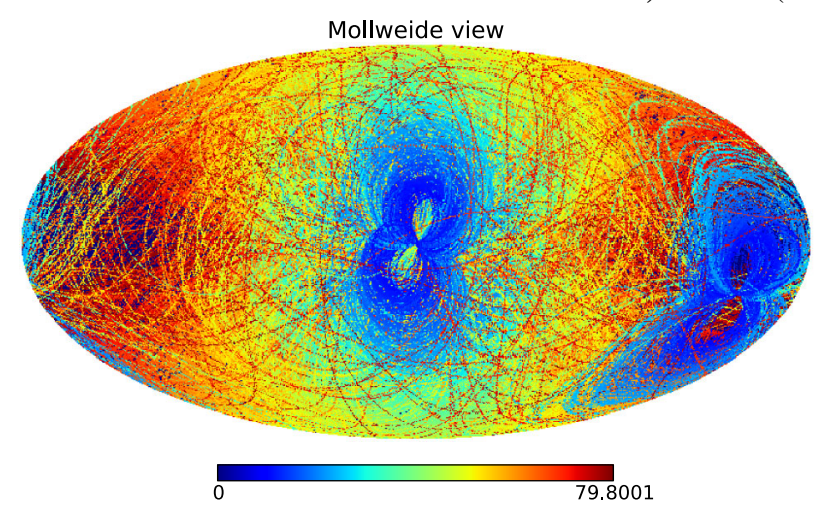

b)

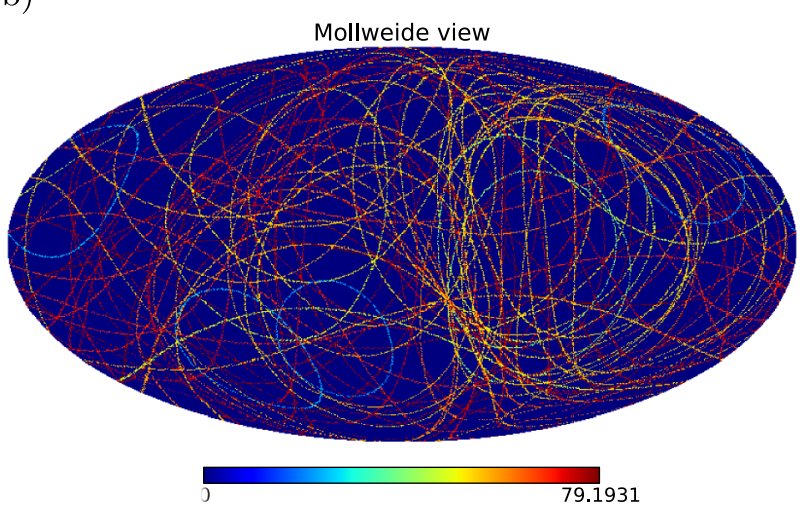

d)

FIG. 10 (color online). For the search for nonorientable manifolds: Location of the circle pairs with maximal statistics $S$ that lies above the $3 \sigma$ C.L., colored by opening angle $\alpha$. Note that the circles that touch either the galactic anticenter or $l \approx 29^{\circ}, b \approx-19^{\circ}$ do not hint at a nontrivial topology. (a) The highest signal comes from circle pairs osculating at the galactic anticenter and at $l \approx 29^{\circ}$, $b \approx-19^{\circ}$. (b) Same as (a) but rotated by $180^{\circ}$ such that the galactic anticenter is in the middle of the plot. (c) Removing circle pairs that touch the galactic anticenter, the highest signal comes from pairs that touch $l \approx 29^{\circ}, b \approx-19^{\circ}$. (d) Removing circles that touch either the galactic anticenter or $l \approx 29^{\circ}, b \approx-19^{\circ}$, no special position on the sky is apparent.

separated by $\theta=50^{\circ}$, with a bump clearly visible around $\alpha=25^{\circ}$. This can be understood as the effect of two circles partially overlapping (see Fig. 6). If two circles are separated by twice their opening angle $\alpha$ they osculate ("kiss"). For orientable manifolds this means that their patterns nearly match up along a segment. This effect is almost independent of the absolute magnitude of the opening angle $\alpha$ : the fraction of the circles that "kiss" is independent of $\alpha$ (up to effects of finite pixel size). However, it is interesting to note that most of the osculating circles that give the maximal $S$ for a given separation angle and opening angle osculate at either of two positions: at the galactic anticenter, or at $l \approx 29^{\circ}, b \approx-19^{\circ}$ (see Fig. 7).

Note that there is no known topology that would lead to such a structure of spikes in the $S$ statistics, correlating the opening angle with the separation angle, confined to just two positions on the sky and appearing both in the search for orientable topologies as well as in the search for nonorientable topologies (see next subsection). Hence it is justified to remove all circles that touch either the galactic anticenter or the position $l \approx 29^{\circ}, b \approx-19^{\circ}$. The existence of this anomaly near the galactic anticenter is perhaps not surprising. The anticenter is in the middle of the ILC portion of the map, and ILC pixels have much more correlated noise properties than the rest of the map. The second point is near the region where the noise properties of the maps are very nonuniform (see Fig. 3 in Ref. [28]).

First excising circles that osculate at the galactic anticenter, Fig. 7(c), and then those that osculate at $l \approx 29^{\circ}$, $b \approx-19^{\circ}$, Fig. $7(\mathrm{~d})$, removes most systematic spikes from the $S$ statistics. The peaks that are left for separation angles $\theta>170^{\circ}$ and opening angle $\alpha \approx 90^{\circ}$ are caused by the fact that circles with $\alpha=90^{\circ}$ have an angular diameter of $180^{\circ}$. Thus, two such circles, when separated by $\theta \approx 180^{\circ}$ will start overlapping, until for $\theta=180^{\circ}$, they coincide, independent of the topology of the Universe.

Hence Fig. 8 presents the final result of the search for orientable topologies. The number of spikes above the $2 \sigma$ and $3 \sigma$ thresholds are consistent with random fluctuations. This leads us to conclude that the data is consistent at $99.7 \%$ C.L. with the null hypothesis of no nontrivial, orientable topology. 


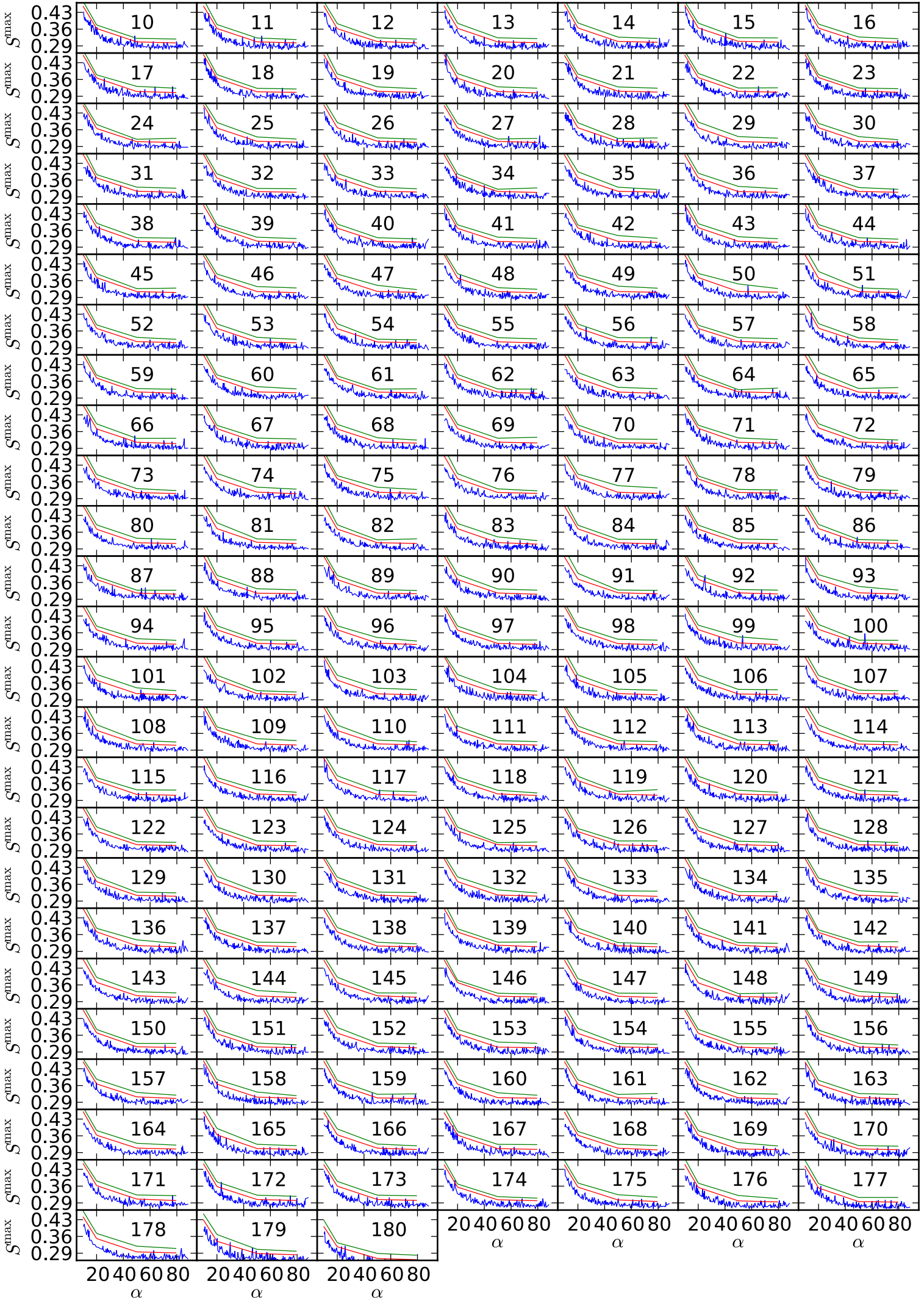

FIG. 11 (color online). Searching for nonorientable topologies, final result: The statistics $S$ (solid blue line) as defined in Eq. (1) as a function of opening angle $\alpha$ and separation angle $\theta$ (indicated by the numbers in the center of each plot). The red (green) line corresponds to the $2 \sigma(3 \sigma)$ C.L. We disregard circles that touch either the galactic anticenter or $l \approx 29^{\circ}, b \approx-19^{\circ}$. No signs of a nontrivial nonorientable topology are found. 


\section{B. Search for nonorientable topologies}

Performing the search for nonorientable topologies on the cleaned CMB map, we again find a multitude of excursions above the $3 \sigma$ C.L. (see Fig. 9). There, we plot in blue the statistics $S$ as a function of opening angle $\alpha$ and separation angle $\theta$ (the former indicated by the numbers in the middle of each panel). The red (green) line is the $2 \sigma$ $(3 \sigma)$ C.L. obtained by scrambling the $a_{\ell m}$ 's as described above. In particular, for low values of the separation angle $\theta$, the signal lies consistently above the $3 \sigma$ C.L. The source of these excursions are again circles that touch either of two distinct spots: the galactic anticenter and $l \approx 29^{\circ}$, $b \approx-19^{\circ}$ [see Fig. 10(a)]. Disregarding all circles that come within $2.5^{\circ}$ of the galactic anticenter, we still find many excursions above the $3 \sigma$ C.L. [see Fig. 10(b)]. Finally, also removing circles that come within $2.5^{\circ}$ of the position $l \approx 29^{\circ}, b \approx-19^{\circ}$, there is no structure left [see Figs. 10(c) and 11].

Figure 11 presents the final, negative result of the search for a nontrivial, nonorientable topology of the Universe. The remaining excursions above $2 \sigma$ and $3 \sigma$ are well explained by random statistical fluctuations as outlined in the previous subsection. This leads us to conclude that the data is consistent at $99.7 \%$ C.L. with the null hypothesis of no nontrivial, nonorientable topology.

\section{CONCLUSIONS AND OUTLOOK}

We employed the circles-in-the-sky statistics first devised in Ref. [3], looking for pairs of matching circles of opening angles $10^{\circ}<\alpha<90^{\circ}$ and separation angles $11^{\circ} \leq \theta \leq 180^{\circ}$. We positioned the circle centers on a grid with $N_{\text {side }}=128$ but computed the statistics on the full $N_{\text {side }}=512 \mathrm{CMB}$ map.

While the WMAP 7-year data brought quite some improvements in the noise of the $S_{i j}^{\max }$ statistics (cf. Fig. 2 in Ref. [3]), we find no hints of a nontrivial topology of the Universe (see Figs. 8 and 11). The new search covered a much wider range of possible topologies, and by extending the search to circles with opening angles as small as $10^{\circ}$, we have extended the previous bound on the size of the
Universe to $98.5 \%$ of the diameter of the last scattering surface, or approximately $26 \mathrm{Gpc}$.

There are systematic effects coming from both members of a circle pair touching either the galactic anticenter or the position $l \approx 29^{\circ}, b \approx-19^{\circ}$ (see Figs. 7 and 10). As these positions appear both when looking for orientable and nonorientable manifolds, they cannot be of topological origin but point towards a contamination of the map at these positions. The galactic anticenter region contains significant amounts of galactic emission. While the ILC maps used in this analysis attempt to remove most of this emission, the correlated residuals are a likely source of contamination in the circle searches.

We are looking forward to the data release of the Planck mission, which will offer an exciting new, sharper view of the surface of last scattering, allowing for a better search of signs of nontrivial topology by removing noise particularly at smaller separation angles $\alpha<30^{\circ}$. Further advances in computing power will enable a search on a full $N_{\text {side }} \geq 512$ grid of circle positions.

\section{ACKNOWLEDGMENTS}

It is a pleasure to thank Jeff Weeks for interesting discussions. Some of the results in this paper have been derived using the HEALPix [29] package. This work made use of the High Performance Computing Resource in the Core Facility for Advanced Research Computing at Case Western Reserve University. We acknowledge the use of the Legacy Archive for Microwave Background Data Analysis (LAMBDA). This work was supported in part by an allocation of computing time from the Ohio Supercomputer Center. Support for LAMBDA is provided by the NASA Office of Space Science. G. D. S. and P. M. V. were supported by a grant from the U.S. Department of Energy to the particle astrophysics theory group at CWRU. P. M. V. was also supported by the office of the Dean of the College of Arts and Sciences, CWRU, and the Impuls und Vernetzungsfond of the Helmholtz Association of German Research Centers under Grant No. HZ-NG-603, and German Science Foundation (DFG) within the Collaborative Research Center 676 Particles, Strings and the Early Universe.
[1] N. J. Cornish, D. N. Spergel, and G. D. Starkman, Classical Quantum Gravity 15, 2657 (1998).

[2] J. J. Levin, Phys. Rep. 365, 251 (2002).

[3] N. J. Cornish, D. N. Spergel, G. D. Starkman, and E. Komatsu, Phys. Rev. Lett. 92, 201302 (2004).

[4] N. J. Cornish, D. Spergel, and G. Starkman, Phys. Rev. D 57, 5982 (1998).
[5] N. J. Cornish, D. N. Spergel, and G. D. Starkman, Proc. Natl. Acad. Sci. U.S.A. 95, 82 (1998).

[6] J.S. Key, N. J. Cornish, D. N. Spergel, and G. D. Starkman., Phys. Rev. D 75, 084034 (2007).

[7] J. Richard Bond, D. Pogosian, and T. Souradeep (unpublished).

[8] J. Richard Bond, D. Pogosian, and T. Souradeep, Classical Quantum Gravity 15, 2671 (1998). 
[9] T. Souradeep, D. Pogosian, and J. Richard Bond, in Proceedings of 33rd Rencontres de Moriond: Fundamental Parameters in Cosmology, Les Arcs, France, 1998 (unpublished).

[10] J. Richard Bond, D. Pogosian, and T. Souradeep, Phys. Rev. D 62, 043005 (2000).

[11] J. Richard Bond, D. Pogosian, and T. Souradeep, Phys. Rev. D 62, 043006 (2000).

[12] M. Lachieze-Rey and J.-P. Luminet, Phys. Rep. 254, 135 (1995).

[13] R. Lehoucq, M. Lachieze-Rey, and J. P. Luminet, Astron. Astrophys. 313, 339 (1996).

[14] J.-P. Luminet and B. F. Roukema, in Proceedings of NATO Advanced Study Institute: Summer School on Theoretical and Observational Cosmology, Cargese, Corsica, France, 1998 (unpublished).

[15] J.-P. Uzan, R. Lehoucq, and J.-P. Luminet, in Proceedings of 19th Texas Symposium on Relativistic Astrophysics: Texas in Paris, Paris, France, 1998 (unpublished).

[16] J.-P. Uzan, R. Lehoucq, and J.-P. Luminet, Astron. Astrophys. 351, 766 (1999).
[17] R. Lehoucq, J.-P. Uzan, and J.-P. Luminet, Astron. Astrophys. 363, 1 (2000).

[18] A. Riazuelo, J. Weeks, J.-P. Uzan, R. Lehoucq, and J.-P. Luminet, Phys. Rev. D 69, 103518 (2004).

[19] J. Weeks, J.-P. Luminet, A. Riazuelo, and R. Lehoucq, Mon. Not. R. Astron. Soc. 352, 258 (2004).

[20] A. Riazuelo, S. Caillerie, M. Lachieze-Rey, R. Lehoucq, and J.-P. Luminet (unpublished).

[21] A. Niarchou and A. H. Jaffe, AIP Conf. Proc. 848, 774 (2006).

[22] A. Niarchou and A. Jaffe, Phys. Rev. Lett. 99, 081302 (2007).

[23] P. Bielewicz and A. J. Banday (unpublished).

[24] P. Bielewicz, A. J. Banday, and K. M. Gorski (unpublished).

[25] M. Frigo and S. G. Johnson, Proc. IEEE 93, 216 (2005).

[26] N. Jarosik, C. L. Bennett, J. Dunkley, B. Gold, M. R. Greason et al., Astrophys. J. Suppl. Ser. 192, 14 (2011).

[27] B. Gold, N. Odegard, J. L. Weiland, R. S. Hill, A. Kogut et al., Astrophys. J. Suppl. Ser. 192, 15 (2011).

[28] C. L. Bennett et al., Astrophys. J. Suppl. Ser. 148, 1 (2003).

[29] K. M. Gorski, E. Hivon, A. J. Banday, B. D. Wandelt, F. K. Hansen, M. Reinecke, and M. Bartelman, Astrophys. J. 622, 759 (2005). 